\title{
Prototype of Beacon-Mobile Application for Medical Appointment Scheduling Management
}

\author{
Harold Ernesto Caceres Zea, Bch, Shirley Romero Solano, Est, Milagros Motta Rondon, Est, Karim Guevara Puente \\ de la Vega, Dra, Eveling Castro Gutierrez, Msc, Cesar Baluarte Araya, Dr. \\ Universidad Nacional de San Agustín de Arequipa,Perú, hcaceresze@unsa.edu.pe, sromeros@unsa.edu.pe, \\ mmotta@unsa.edu.pe,kguevarap@unsa.edu.pe,ecastro@unsa.edu.pe, cbaluarte@unsa.edu.pe
}

\begin{abstract}
One of the frequent situations which arise in a health center, which creates many inconveniences to the users of health services, is the process of appointments to the various specialized issue. Although it has been designed and implemented many channels of care to obtain appointments, this is still a crucial problem; on the other hand, it is known that the use of mobile devices becomes more usually in the daily activities of the people. In this paper we propose the prototype of a mobile application that allows users a fast and autonomous way to obtain an appointment in the speciality that they require using external devices called Beacons with Bluetooth Low Energy technology, as well as, manage more efficiently resources are there in the health center.
\end{abstract}

Keywords-- Beacon, Mobile Application, Medical Appointment, Scheduling Management.

\section{INTRODUCTION}

Over the years, the problem about to appointment scheduling at hospitals have been increasing, the large queues create a nuisance in patients, that they are mostly adults. Like an evidence, there are some statistics data of the survey in [9]. Health Beacon Appointment application use Bluetooth Low Energy (BLE) with externals devices like Estimote Beacon in order to be used to make appointments in a hospital.

The application will use BLE to connect with Estimote Beacons located in some areas inside the hospital. It will allow patients to make appointments with the information in real time. The application will use an administration system that it will provide the hospital data.

This paper seeks to begin the development of the proposal raised previously, explaining the methods and technologies used to the prototype, requirements and the architecture.

\section{RelATED Works}

Smartphones have changed the life of everyone. Along with other features, an App allows us to do almost everything. Also, when you develop Apps you will face various challenges associated with the design and resources management [1].
Talking the problem of indoor localization is an important next step in the future of the Internet of Things (IoT). With effective indoor localization, we can determine where someone is within a building with reasonable level of accuracy. Museum and historical sites, for example, are already starting to use beacon technology to enrich a visitor's experience by providing them with extra information when they stand in front of an exhibit [2].

Noguchi, Shota, et al. [3] proposed a system which solves the conventional problem of attendance to classes which takes a long time to scan student's cards with the system that it uses a single terminal device. They used a BLE (Bluetooth Low Energy) beacon device to transmit a magic number necessary for proper registration within the classroom.

In Indian, an application was created that used BLE beacon device to collect the data from the sensors and store it according to the dates. This provides a way for the teacher to instantly record and analyze the attendance of all the students. Methods of avoiding proxies or false attendances have also been incorporated [4].

A distributed system was development for collecting radio fingerprints by mobile devices with the Android operating system. This system enables volunteers to create radio-maps and update them continuously. New BLE beacon devices have been installed on the floor of the building in addition to existing $\mathrm{WiFi}$ access points. The localization of stationary objects based on WiFi, Bluetooth Low Energy, and their combination has been evaluated using the data measured during the experiment in the building [5].

As GPS doesn't work indoor of buildings, so that generate some problems, that is the reason the paper presents a method applicable for the purpose of internal navigation using a combination of Beacon transmitters and mobile sensors. This approach has two main stages: a) The first step deals with calculating the user's localization in the building using trilateration, respectively multilateration and b)The second stage of the method serves to validate the first part by using

Digital Object Identifier (DOI):

http://dx.doi.org/10.18687/LACCEI2019.1.1.145

ISBN: 978-0-9993443-6-1 ISSN: 2414-6390

$17^{\text {th }}$ LACCEI International Multi-Conference for Engineering, Education, and Technology: "Industry, Innovation, And Infrastructure for Sustainable Cities and Communities", 24-26 July 2019, Jamaica. 
mobile sensors. They experimented with different input parameters to achieve higher accuracy [6].

A demo used Bluetooth Low Energy (Beacons) and single board computers to track patients in the emerging field of smart care facilities. Our model uses a fixed scanner moving transmitter method for wireless tracking of patients through the facility. The data collected by all scanners is stored within a central database that is designed to be efficiently queried. We show how inexpensive components in conjunction with free open source software can be used to implement a patient tracking system. We focus on the pipeline between acquisition and display of the location data. Additionally, we also discuss the manipulation of the data required for usability, and optional filtering operations that improve accuracy [7].

In order to realize the indoor positioning with these communication systems, techniques such as fingerprinting, trilateration, and triangulation have been widely studied. In the paper [8], they discuss one of these methods used for indoor positioning, i.e., weighted centroid localization (WCL) using received signal strength indicator (RSSI) observed from neighboring BLE beacons. The WCL is evaluated in their testbed building.

The recent introduction of the Bluetooth Low Energy (BLE) radio protocol provides new opportunities for indoor location. It supports portable battery-powered beacons that can be easily distributed at low cost, giving it distinct advantages over WiFi. However, its differing use of the radio band brings new challenges too. It shows the choice of key parameters in a BLE positioning system, including beacon density, transmit power, and transmit frequency. The results show advantages to the use of BLE beacons for positioning [9].

The suggested system automatically uses the Bluetooth 4.0 communication of the student's smartphones when the student go to the lecture hall of the course to check the location of the Beacon and automatically acknowledges attendance if it is valid location. It is characterized by the fact that there is no action to be taken on the part of the student or professor for checking attendance. Also the system recognizes the student and professor modes using the smart phones number and registers students and professors [10].

In [11] the proposal was a new system framework using BLE Beacon to detect the user location and conduct power management in the home through a mobile device application. Due to the BLE Beacon may produce the multipath effect, this study uses the positioning algorithm and hardware configuration to reduce the error rate. Location fingerprint positioning algorithm and filter modification were used to establish a positioning method for facilitating deployment and saving computing resources.
The paper [12] propose an algorithm that uses the combination of channel-separate polynomial regression model (PRM), channel-separate fingerprinting (FP), outlier detection and extended Kalman filtering (EKF) for smartphone-based indoor localization with BLE beacons.

\section{MateRials AND METHODS}

\section{A. Scenarios}

The prototype of this paper should be easy to use, enjoyable and could be applied to design different applications for the different purposes (scenarios): a) A crowded scenario of patients in a hospital, where the users need to reach an appointment in a short time without lost a lot of time making queues. How would users make an appointment in real time?, b) the people don't have respectful to others who are waiting for a long time and they can make an appointment before others who arrived early than them. How they can make an appointment in least of one minute?, c) an scenario of parents that they have to make an appointment for their child, and d) make an appointment and go to the doctor office. How to improve the whole process?

To achieve the previously mentioned scenarios; a clear, an tradicional software development phases should be followed: Analysis, Design, Implementation and Testing.

\section{B. Flowchart}

The main purpose of this work is to show the analysis and design Health Appointment Beacon application software by name of HealthBeacon to be able to use it as a support in the process of planning and obtaining medical appointments.

The flowchart of the HealthBeacon Application is shown in figure 1. First, the patients need to install the application in their mobiles. Then the App check whether the Bluetooth is "on" or "off". If the bluetooth is "on" then the user will receive the information of the specialities available for the current day depending on which beacon is nearest to the user. If the user is near to Beacon 1 then the smartphone will display all specialities associated to this beacon due to each beacon has different specialities registered. If the user is not near any of beacons the user won't see the specialities in order to make an appointment.

After that, if the user walks to other position of the beacon, it will display the information related to that beacon or it will just show a "Not specialities available" message. In this way, the user needs to have an internet connection due to the administration server is on the web and the App needs to make request to this system. 


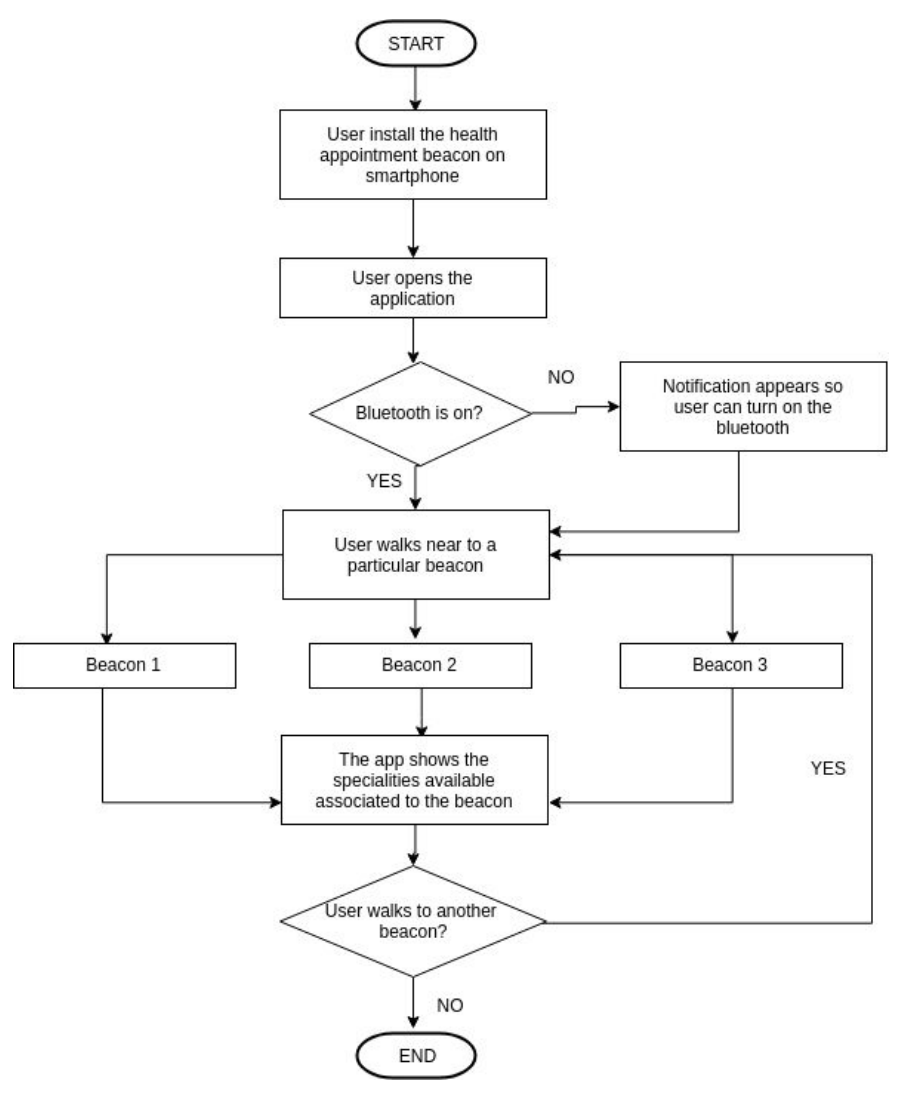

Fig. 1 FlowChart HealthBeacon Application.

\section{Bluetooth Low Energy and Estimote Beacons}

The advent of Bluetooth Low Energy has opened up new possibilities in the past few years for applications to take advantage of micro locations capabilities. Bluetooth Low Energy (BLE) is an inexpensive and energy-efficient version of Classic Bluetooth communication standard that has made communication between nearby devices more feasible than ever before, greatly empowering advances in the Internet of Things.

Bluetooth Low Energy (BLE) is gaining popularity as a convenient way of wireless communication due to its slow power requirement and inexpensive characteristic compared with classic Bluetooth. Likewise, Estimote Beacons are small transmitters, run on BLE technology, used as point of reference for mobile devices and they can detect a Bluetooth enabled device when it is in its transmission range. In this work, for testing purpose, we are using Estimote Beacons. Estimote give us a library to recognize beacons signals inside the application. The table I lists the features of Estimote beacons.
TABLE I

Estimote Beacons Features.

\begin{tabular}{|c|c|}
\hline Properties & Specification \\
\hline Battery Life & $<=5$ years \\
\hline Range & $<=100$ Meters \\
\hline Fleet management & Estimote SDK \\
\hline Built-in radios & Bluetooth 5.0 \\
\hline
\end{tabular}

We opted to purchase from Estimote due to their support for multiple protocols, affordable pricing, convenient developer tools, and well-supported, active developer forums. Therefore, we configured the beacons using Estimote App for Android and the Estimote Cloud web application. We need to consider that transmitting power determines how strongly the BLE signal from the beacon is sent, affecting the range where devices will be able to detect the beacom. So, transmitting at larger distance consumes more power, so adjusting this setting will also affect the beacon's projected battery life.

The figure 2 shows a screenshot of Estimote's configuration app. If you update some features in the Estimote's web, you need to open the app in order to apply those changes to the beacon.

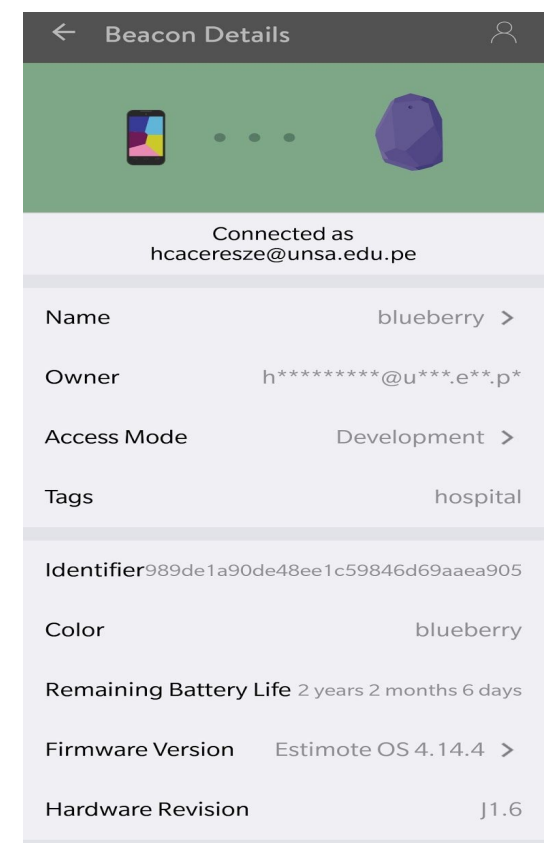

Fig. 2 Estimote App display the settings one can modify after selecting a beacon to configure.

The next steps would be to create tags-based identification, it means, define your proximity zones with human-readable tags instead of abstract identifiers. For our

$17^{\text {th }}$ LACCEI International Multi-Conference for Engineering, Education, and Technology: "Industry, Innovation, And 
application we grouped them into "hospital". Knowing when the user enters range of beacon " $1 \mathrm{~b} 4 \mathrm{fe}$ " is not that useful in and on itself. However, if your App somehow knows that beacon " $1 \mathrm{~b} 4 \mathrm{fe}$ " is placed on specific place, we can manage custom message in range of beacon " $1 \mathrm{~b} 4 \mathrm{fe}$ " suddenly means close to emergency module inside the hospital.

In simple terms, most of the time, proximity beacons only makes sense if you can give your beacons some meaning. Estimote allow us to attach some extra data/meaning to a beacon in order to manage better the identification inside the healthbeacon application. In our configuration we set up two beacons with attachment differentes with the key "location" for both. The figure 3 shows the code to identify only the beacons with the tag "hospital" and we get the value from location key inside attachment.

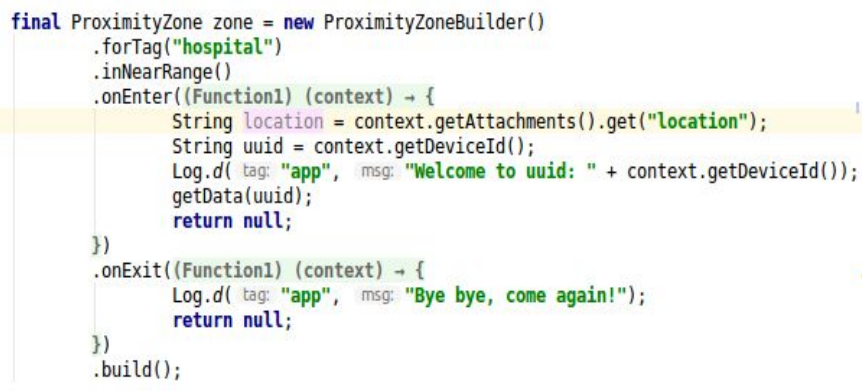

Fig. 3 The above code demonstrates how to recognize a beacon by tag and get its attachment.

As you refer [8], the use of this type of BLE beacon offers more precision in indoor localization compared to other existing technologies within mobile devices e.g., GPS or WiFi.

\section{The Smartphones' Operating System}

The Android operating system is an open source and free platform; it is not bound to one hardware provider or manufacturer. This openness of Android is allowing a quick gain of the market share. For developing of our application we chose android as platform.

\section{E. Language programming and Frameworks}

1) Python: Is a high-level, interpreted and general-purpose dynamic programming language that focuses on code readability. We chose this language due to extensive support libraries and integration feature.

2) Django and Django Rest Framework: This framework encourage rapid development and clean, pragmatic design to create a faster system administration. Furthermore, we developed and API to create the services to connect with the mobile application (client).

\section{F. Development Application}

The aim of this work is to provide to patients an application to make appointments inside a hospital. In this paper, we present a system administration and mobile application. The application recognize beacons signal and show up the available specialities for the current day. The figure 4 shows a screenshot of register page to allow the users obtain a username and password. Beside, the main menu inside the app with the current options of the application. The administration system allows to manage all the data to show in the application. The figure 5 shows the dashboard of administration system.

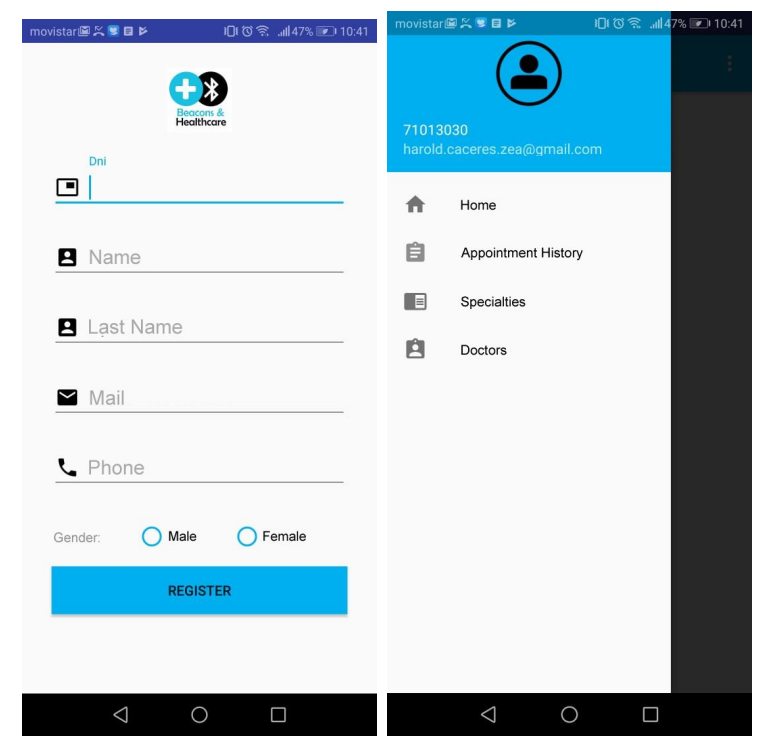

Fig. 4 Register and Menu screenshot of application.

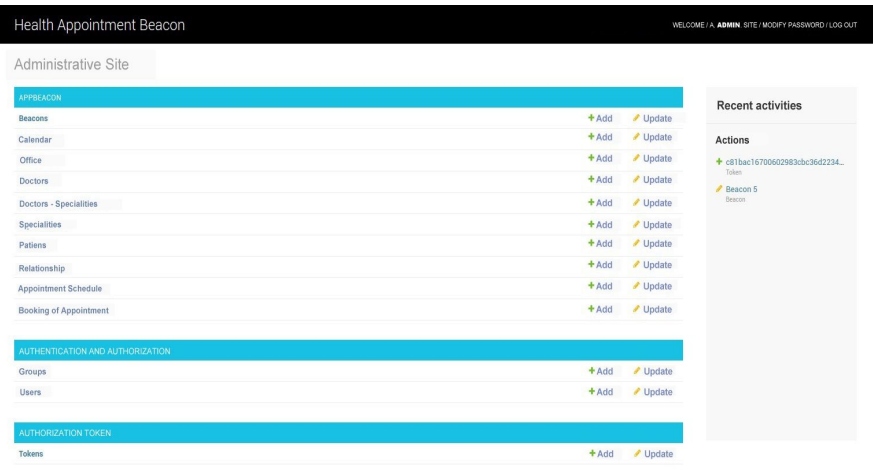

Fig. 5 System web administration dashboard.

G. Analysis - Requirements:

1. Functional Requirements:

Administration System

- As a user, we want to register specialities

$17^{\text {th }}$ LACCEI International Multi-Conference for Engineering, Education, and Technology: "Industry, Innovation, And 
- As a user, we want to register doctor profile with their specialities

- As a user, we want to register doctor office

- As a user, we want to register beacons information

- As a user, we want to register appointments scheduling

- As a user, we want to register patients with basic information

Android Application

- As a user, we want to register

- As a user, we want to see a list with the available appointments

- As a user, we want to make an appointment

\section{Non-Functional Requirements:}

- The application should be installed in android smartphone version greater than 7 .

- The mobile smartphone needs to have bluetooth adapter.

- The application should have internet connection in order to communicate with system administration.

\section{H. Diseño}

1. Architecture:

The figure 6 shows the architecture diagram used to development web system administration. We are running backend in the server. It has an API to manage all the request from mobile application. We have postgres database to manage the data from application and web system as well.

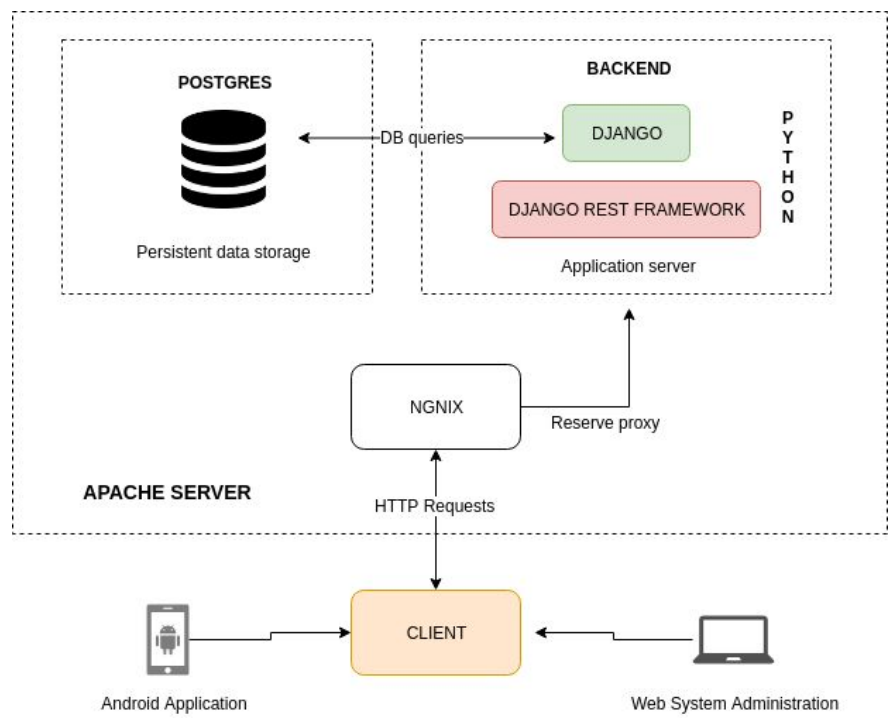

Fig. 6 Architecture diagram.

This architecture allow us create more than one client to make request to API. For now, we only have a mobile application using the API endpoints.

\section{Database:}

$1^{\text {th }}$ LACCEI International Multi-Conference for Engineering, Education, and Technology: “Industry, Innovation, And Infrastructure for Sustainable Cities and Communities", 24-26 July 2019, Jamaica.
We chose Postgres Relational Database due to space and time efficiency. The data is organized in several tables, but for showing the list specialities available, we have four tables. One represents the beacon data like UUID, name and location. The second table represents the specialities, it has information related to speciality. The third table has many to many relationship between specialities and beacons tables, so one speciality could be have more than one beacon associated. The last table has the scheduling appointments for the current day.

The figure 7 shows the relationship between these tables. Also we have other tables to manage the doctors and the relationship between specialities.

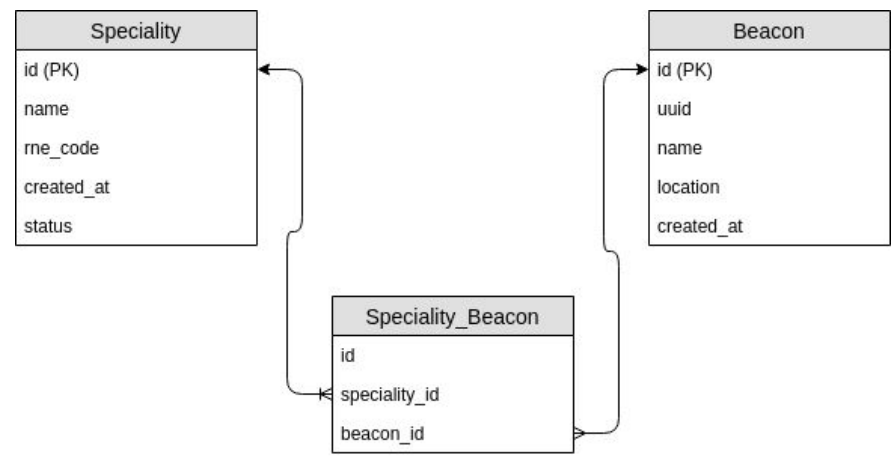

Fig. 7 The above tables represents the relationship between speciality and beacon. Note that there is a table to manage many to many relationship between them.

The figure 8 shows the tables users and tokens as part of security module. These tables save the token allowing application users to connect with the system and retrive data. Each users created through application generate a new token.

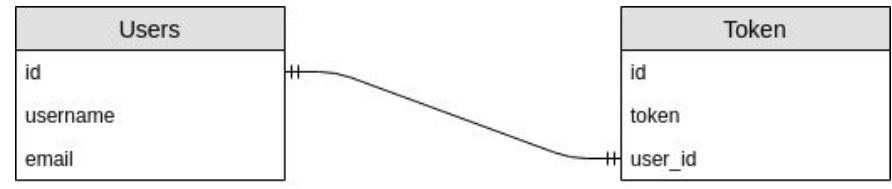

Fig. 8 The tables above table represents the relation between user and token to give user access to mobile application.

The system administration allows all the tables to be managed in the postgres database manager.

\section{DISCUSSION}

In the previous paper [1], we have chosen php and Mysql to development the application. We selected the right language programming and database that they allow us develop new features inside the application. In this scenario, we needed to select a framework extremely fast, scalable secure and easy-to-use that allow us to create new functionalities in a scalable way. Python has an exceptionally well-developed libraries support for almost types of applications. 
Other change that we did is the database engine. We have chosen postgres instead of mysql. Postgres is widely used in large systems where read and write speeds are crucial and data needs to validated. In addition, it supports a variety of performance optimizations. Overall, postgres performance is utilized best in systems requiring execution of complex queries.

In [1] the beacons used was "jalee", but the library to manage the connections was deprecated. So, for this reason we decided to search other options in beacons brand. We chose Estimote due to the company is in constantly developing to offer better products. Likewise, the new product released at 2018 integrated Bluetooth 5.0 standard including mesh networking, long range, and high data throughput.

\section{RESULTS}

We have tried the application in an environment with two beacons with differents distances to the user. The figure 9 shows the scenario.

Each beacons has associated different specialities, the application will recognize nearer beacon to the user and show the list of specialities in the figure 10. We can see different available specialities for each beacon. Then, the user will be able to select the specialities and he will see other list with the doctors available and the maximum number of appointments for each one such as in the figure 11.

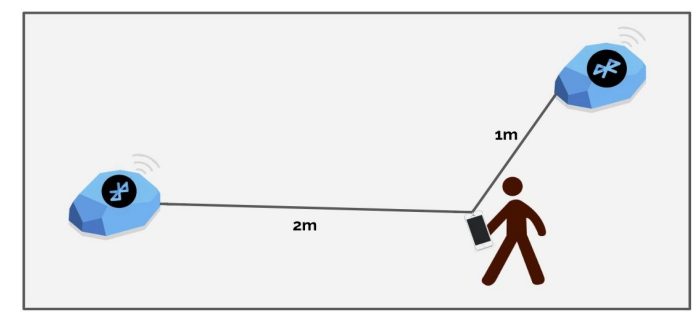

Fig. 9 Scenario to test the signals beacons
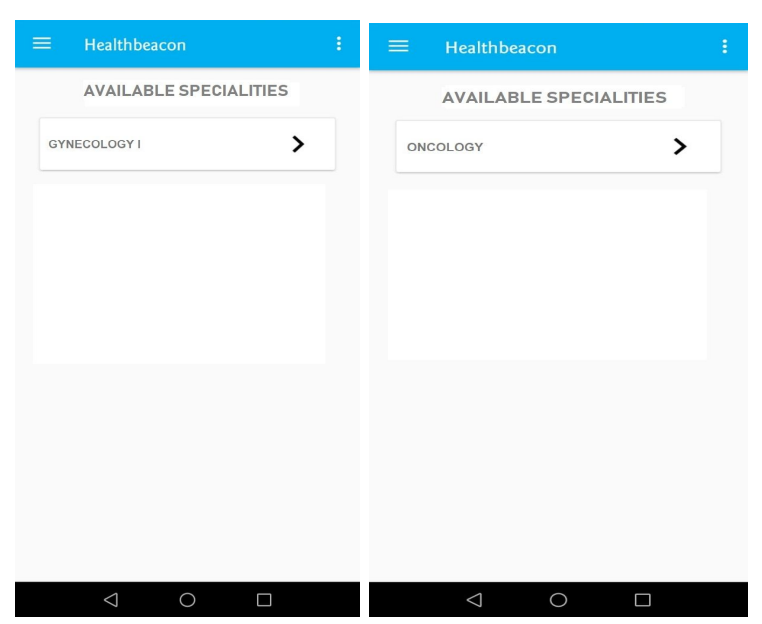

Fig. 10 Prototype of list specialities available for different beacons represented in the figure 9 .
We have tested the application in the follow context: One user was between the range 2 meters to beacon 1 and the second user was between the range 1 meter to beacon 2. Both, could get the specialities availables for each. Likewise, we measured the response time in the application until get the list of specialities. We observed that we can attend more than 10 user by minute due to the beacon signals were emitted each $200 \mathrm{~ms}$. One minute has 60000 , so we will be able to emit 600 signals by minute. The logic of the application is recognize the nearest beacon signals to the user. So, in our scenario the user will be able to recognize of beacon separated by 1 meter.

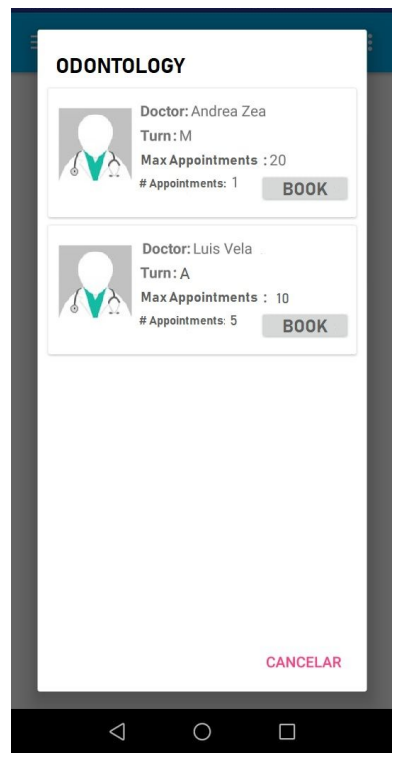

Fig. 11 Prototype of doctor's list available for the speciality selected.

\section{CONCLUSIONS}

This paper presented healthbeacon. This app utilizes the Bluetooth Low Energy (BLE) technology with external devices that utilizes Bluetooth, namely beacons. The proposed recognition beacons signal can also be used in other applications such as stadium, offices, retails and universities. For experimentation, beacon were place in a place and use the healthbeacon app to make appointments which detect the beacons signals. The results shows that when the mobile come in the range of beacon, it shows specialities associated in this beacons on the smartphone screen. This application would take the advantage to allow a many user make an appointments in the same time. The system manage the concurrency in the request from each user. In less one minute the system will be able to response many request and show the real data to the other users.

\section{FUTURE WORKS}

$17^{\text {th }}$ LACCEI International Multi-Conference for Engineering, Education, and Technology: "Industry, Innovation, And Infrastructure for Sustainable Cities and Communities", 24-26 July 2019, Jamaica. 
In order to obtain a scalable system for the future, there are several aspects which could be improved, follow three of them: a) create a service for running in the background to detect beacon signals without expend a lot of battery in the mobile, b) create an algorithm to manage the recognition of the beacons signals in a better way when there will be more than 2 beacons at the same distance of the user, c) create a software to process and analyse data generated for the users in order to make a predictions and make a decisions in real time without waste a lot of time analysing data manually.

\section{ACKNOWLEDGMENT}

This research project was subsidized by Universidad Nacional de San Agustín de Arequipa, IAI-023-2018-UNSA. We would like to thank to the "Research Center, Transfer of Technologies and Software Development R + D + i" CIDETI-CiTeSoft-UNSA for their collaboration in the use of their equipment and facilities, for the development of this research work.

\section{REFERENCES}

[1] H. Caceres, E. Castro-Gutierrez, Development of a Mobile Platform for Appointment Management of a Hospital Using Bluetooth Low Energy Technology with External Devices - Beacons, Innovation in Education and Inclusion: Proceedings of the 16th LACCEI International Multi-Conference for Engineering, Education and Technology, July $18-20,2018$

[2] A.Budan, A. Naderi and L Deugo, Range of Bluetooth Low Energy Beacons in Relation to Their Transmit Power, School of Computer Science, Carleton University, Canada, 2017.

[3] Noguchi, Shota, et al. Student attendance management system with bluetooth low energy beacon and android devices. Network-Based Information Systems (NBiS), 2015 18th International Conference on. IEEE, 2015.

[4] Apoorv, R., \& Mathur, P. (2016, November). Smart attendance management using Bluetooth Low Energy and Android. In Region 10 Conference (TENCON), 2016 IEEE (pp. 1048-1052). IEEE.

[5] Kriz, P., Maly, F., \& Kozel, T. (2016). Improving indoor localization using bluetooth low energy beacons. Mobile Information Systems, 2016.

[6] M. Liskovec, A. Kovarova: Beacon Based Localization Refined by Outputs from Mobile Sensors, CompSysTech 2016, ACM, to be published.

[7] Kennedy, B., Taylor, G. W., \& Spachos, P. (2018, March). BLE Beacon Based Patient Tracking in Smart Care Facilities. In 2018 IEEE International Conference on Pervasive Computing and Communications Workshops (PerCom Workshops) (pp. 439-441). IEEE.

[8] Subedi, Santosh \& Kwon, Goo-Rak \& Shin, Seokjoo \& Hwang, Suk-seung \& Pyun, Jae-Young. (2016). Beacon based indoor positioning system using weighted centroid localization approach. 1016-1019. 10.1109/ICUFN.2016.7536951.

[9] R. Faragher and R. Harle, "Location Fingerprinting With Bluetooth Low Energy Beacons," in IEEE Journal on Selected Areas in Communications, vol. 33, no. 11, pp. 2418-2428, Nov. 2015.doi: 10.1109/JSAC.2015.2430281

[10] Mi-Young Bae, Dae-Jea Cho. (2015). Design and Implementation of Automatic Attendance Check System Using BLE Beacon. International Journal of Multimedia and Ubiquitous Engineering, 10(10), 177-186.

[11] Ke, C. K., Ho, W. C., \& Lu, K. C. (2018, October). Developing a Beacon-based Location System Using Bluetooth Low Energy Location
Fingerprinting for Smart Home Device Management. In International Wireless Internet Conference (pp. 235-244). Springer, Cham.

[12] Yuan Zhuang 1, Jun Yang 1, , You Li 2,3, Longning Qi 1 and Naser El-Sheimy, (2016), Smartphone-Based Indoor Localization with Bluetooth Low Energy Beacons, doi:10.3390/s16050596.

$1^{\text {th }}$ LACCEI International Multi-Conference for Engineering, Education, and Technology: “Industry, Innovation, And Infrastructure for Sustainable Cities and Communities”, 24-26 July 2019, Jamaica. 